\title{
Early pregnancy vitamin D status and risk of preeclampsia
}

\begin{abstract}
Hooman Mirzakhani, ${ }^{1}$ Augusto A. Litonjua, ${ }^{1,2}$ Thomas F. McElrath, ${ }^{3}$ George 0 'Connor, ${ }^{4}$ Aviva Lee-Parritz, ${ }^{5}$ Ronald Iverson, ${ }^{5}$ George Macones, ${ }^{6}$ Robert C. Strunk, ${ }^{7}$ Leonard B. Bacharier, ${ }^{7}$ Robert Zeiger, ${ }^{8}$ Bruce W. Hollis, ${ }^{9}$ Diane E. Handy, ${ }^{10}$ Amitabh Sharma, ${ }^{1}$ Nancy Laranjo, ${ }^{1}$ Vincent Carey, ${ }^{1}$ Weilliang Qiu, ${ }^{1}$ Marc Santolini, ${ }^{111}$ Shikang Liu, ${ }^{12}$ Divya Chhabra, ${ }^{13}$ Daniel A. Enquobahrie, ${ }^{14}$ Michelle A. Williams, ${ }^{15}$ Joseph Loscalzo, ${ }^{16}$ and Scott T. Weiss ${ }^{1,17}$

1'Channing Division of Network Medicine, Department of Medicine, ${ }^{2}$ Division of Pulmonary and Critical Care Medicine, Department of Medicine, and ${ }^{3}$ Division of Maternal-Fetal Medicine, Department of Obstetrics and Gynecology, Brigham and Women's Hospital, Harvard Medical School, Boston, Massachusetts, USA. Pulmonary Center, Department of Medicine, Boston Medical Center, Boston University, Boston, Massachusetts, USA. ${ }^{5}$ Department of Obstetrics and Gynecology, Boston Medical Center, Boston University, Boston, Massachusetts, USA. ${ }^{6}$ Division of Maternal-Fetal Medicine, Department of Obstetrics and Gynecology, and 'Division of Pediatric Allergy, Immunology and Pulmonary Medicine, Department of Pediatrics, Washington University, St. Louis, Missouri, USA. ${ }^{8}$ Department of Allergy and Research and Evaluation, Kaiser Permanente, San Diego, California, USA. ${ }^{9}$ Department of Pediatrics, Medical College of South Carolina, Charleston, South Carolina, USA. ${ }^{10}$ Division of Cardiovascular Medicine, Department of Medicine, Brigham and Women's Hospital, Boston, Massachusetts, USA. "'Center for Complex Networks Research (CCNR), Northeastern University, Boston, Massachusetts, USA. ${ }^{12}$ Center for Interdisciplinary Research on Complex Systems, Department of Physics, Northeastern University, Boston, Massachusetts, USA. ${ }^{13}$ Division of Pediatric Pulmonary, Department of Pediatrics, University of California, San Diego, California, USA. ${ }^{14}$ Department of Epidemiology, School of Public Health, University of Washington, Seattle, Washington, USA. ${ }^{15}$ Department of Epidemiology, Department of Clobal Health and Populations, Harvard T.H. Chan School of Public Health, Boston, Massachusetts, USA. ${ }^{16}$ Department of Medicine, Brigham and Women's Hospital, Harvard Medical School, Boston, Massachusetts, USA. ${ }^{17}$ Partners Center for Personalized Medicine, Partners Health Care, Boston, Massachusetts, USA.
\end{abstract}

BACKGROUND. Low vitamin D status in pregnancy was proposed as a risk factor of preeclampsia.

METHODS. We assessed the effect of vitamin D supplementation (4,400 vs. 400 IU/day), initiated early in pregnancy (10-18 weeks), on the development of preeclampsia. The effects of serum vitamin D (25-hydroxyvitamin D [250HD]) levels on preeclampsia incidence at trial entry and in the third trimester (32-38 weeks) were studied. We also conducted a nested casecontrol study of 157 women to investigate peripheral blood vitamin D-associated gene expression profiles at 10 to 18 weeks in 47 participants who developed preeclampsia.

RESULTS. Of 881 women randomized, outcome data were available for 816 , with 67 (8.2\%) developing preeclampsia. There was no significant difference between treatment $(N=408)$ or control $(N=408)$ groups in the incidence of preeclampsia $(8.08 \%$ vs. $8.33 \%$, respectively; relative risk: $0.97 ; 95 \% \mathrm{Cl}, 0.61-1.53)$. However, in a cohort analysis and after adjustment for confounders, a significant effect of sufficient vitamin $D$ status $(250 \mathrm{HD} \geq 30 \mathrm{ng} / \mathrm{ml})$ was observed in both early and late pregnancy compared with insufficient levels $(250 \mathrm{HD}<30 \mathrm{ng} / \mathrm{ml}$ ) (adjusted odds ratio, $0.28 ; 95 \% \mathrm{Cl}, 0.10-0.96)$. Differential expression of 348 vitamin D-associated genes (158 upregulated) was found in peripheral blood of women who developed preeclampsia (FDR $<0.05$ in the Vitamin D Antenatal Asthma Reduction Trial [VDAART]; $P<\mathbf{0 . 0 5}$ in a replication cohort). Functional enrichment and network analyses of this vitamin D-associated gene set suggests several highly functional modules related to systematic inflammatory and immune responses, including some nodes with a high degree of connectivity.

CONCLUSIONS. Vitamin D supplementation initiated in weeks 10-18 of pregnancy did not reduce preeclampsia incidence in the intention-to-treat paradigm. However, vitamin D levels of $30 \mathrm{ng} / \mathrm{ml}$ or higher at trial entry and in late pregnancy were associated with a lower risk of preeclampsia. Differentially expressed vitamin D-associated transcriptomes implicated the emergence of an early pregnancy, distinctive immune response in women who went on to develop preeclampsia.

TRIAL REGISTRATION. ClinicalTrials.gov NCT00920621.

FUNDING. Quebec Breast Cancer Foundation and Genome Canada Innovation Network. This trial was funded by the National Heart, Lung, and Blood Institute. For details see Acknowledgments.

\section{Related Commentary: p. 4396}

Authorship note: R.C. Strunk is deceased.

Conflict of interest: The authors have declared that no conflict of interest exists. Submitted: June 13, 2016; Accepted: September 16, 2016.

Reference information: J Clin Invest. 2016;126(12):4702-4715. doi:10.1172/JCI89031.

\section{Introduction}

Preeclampsia is a multisystem complex disease of pregnancy with grave maternal-fetal and neonatal consequences that complicates up to $8 \%$ of pregnancies $(1,2)$. Low vitamin $\mathrm{D}$ status, among other risk factors, is linked to the development of preeclampsia. Systematic reviews and meta-analyses have concluded that low serum 
vitamin D levels (25-hydroxyvitamin D [25OHD]) in pregnancy are associated with a higher risk of preeclampsia and suggest a preventive role of vitamin D supplementation (3-6). However, a recent meta-analysis of the 3 vitamin D supplementation randomized clinical trials (RCTs) on pregnancy outcomes found no association between increased serum vitamin D levels and preeclampsia risk (7). Although these studies vary in the dose of vitamin D supplementation (400-4,000 IU/day) and in the baseline and attained vitamin D levels, it is most important to note that all of the RCTs started the intervention after 20 weeks of gestation. It is noted that this meta-analysis did not include the results of the National Institute of Child Health and Development (NICHD) trial that started vitamin D supplementation before 20 weeks of gestation (8). A meta-analysis of the abnormal maternal biomarkers found during the first trimester of pregnancy in women who developed preeclampsia and the findings of previous studies on the association of preeclampsia and low vitamin D status in early pregnancy suggest the necessity of early serum vitamin D level surveillance and modification in pregnancy $(5,9)$. As such, our trial, the Vitamin D Antenatal Asthma Reduction Trial (VDAART), investigated the effect of early pregnancy vitamin D supplementation and both early and late pregnancy 25OHD levels on the risk of preeclampsia, one of the a priori-defined outcomes of the trial.

Previous investigations have also shown the effect of vitamin D levels on peripheral blood gene expression during pregnancy and in preeclampsia $(10,11)$. Global gene expression profiling has the potential to identify representative pathway genes that might point to the underlying molecular mechanism of preeclampsia (12-15). Therefore, we performed a nested case-control study in the VDAART to identify vitamin D gene signatures related to preeclampsia during early pregnancy. There is increasing evidence that disease signature and causal genes do not distribute randomly on the molecular interaction network (interactome), but rather interact in similar biological pathways $(16,17)$. Accordingly, we applied a molecular interaction network approach using information from differential expression and a protein-protein interaction network to identify interacting key drivers (preeclampsia module) of the vitamin D gene signatures.

\section{Results}

Low vitamin D status in early pregnancy and throughout is associated with a higher risk of preeclampsia. Of 9,268 women who were screened, 881 were randomized. After exclusion of 5 ineligible women, 440 were assigned to receive vitamin D and 436 to receive placebo (Figure 1). A total of 32 women in the treatment group and 28 in the placebo group (60 of $876,6.8 \%$ overall) were lost to follow-up.

Among the baseline characteristics, gravidity was significantly different between the 2 study groups (Table 1). Vitamin D levels during the third trimester in the treatment group amounted to 39 $\pm 15 \mathrm{ng} / \mathrm{ml}$ versus $27 \pm 11 \mathrm{ng} / \mathrm{ml}$ in the placebo group $(P<0.0001)$. No side effects or adverse events were reported in association with vitamin D supplementation. Preeclampsia occurred in 33 women in the treatment group (8.08\%) as compared with 34 women in the placebo group (8.33\%) (relative risk [RR], 0.97; 95\% CI, 0.61-1.53). While vitamin D supplementation increased 25OHD levels in the third trimester, no reduction in the rate of preeclampsia was seen in the vitamin D treatment group as compared with that in the placebo group. However, women who had sufficient vitamin D levels (at least $30 \mathrm{ng} / \mathrm{ml}$ ) in both early and late pregnancy, regardless of treatment group, showed a significantly lower incidence of preeclampsia as compared with those who had an insufficient level at these time points $(2.25 \%$ vs. $11.92 \%$; RR, 0.20 ; CI, $0.06-0.66 ; P<$ $0.008)$. Within the treatment and placebo control groups, trends toward a decreased occurrence of preeclampsia were observed among women who had sufficient vitamin D status $(\geq 30 \mathrm{ng} / \mathrm{ml})$ in both early and late pregnancy (high-high 25OHD: odds ratio [OR], 0.6; CI, 0.35-1.06; $P=0.08$ and OR, 0.35; CI, 0.16-0.78; $P=0.01$, respectively). The pooled estimate of the OR in the intention-totreat (ITT) population was 0.55 (CI, 0.37-0.82; $P<0.003)$. After covariate adjustment (Table 2), women with sufficient vitamin D concentrations at both 10 to 18 and 32 to 38 weeks of gestation had a substantially reduced risk of preeclampsia (adjusted OR, 0.28; CI, 0.08-0.96; $P=0.04)$. 25OHD serum concentrations in early pregnancy were a predictor of preeclampsia (adjusted OR, 0.96; CI, 0.93-0.99; $P=0.025$ ), such that a 10-, 20-, or 30-unit increase $(\mathrm{ng} / \mathrm{ml})$ was associated with a $33 \%, 54 \%$, and $70 \%$ reduction in the risk of preeclampsia, respectively (adjusted $\mathrm{OR}_{10 \text {-unit }}=0.67$; 95\% CI, 0.47-0.95; adjusted $\mathrm{OR}_{20 \text {-unit }}=0.46$; 95\% CI, 0.22-0.90; adjusted $\mathrm{OR}_{30 \text {-unit }}=0.30 ; 95 \% \mathrm{CI}, 0.16-0.85$, respectively). No occurrence of preeclampsia was noted in women with a baseline serum value above $37 \mathrm{ng} / \mathrm{ml}$. Accordingly, the adjusted estimated probability of preeclampsia was less than 0.05 at an approximate vitamin D serum level of $40 \mathrm{ng} / \mathrm{ml}(100 \mathrm{nmol} / \mathrm{l})$ during early pregnancy (Figure 2).

Early pregnancy vitamin D status modulates the expression of preeclampsia transcriptome signatures. Marginal comparisons of distributions of maternal age, race, and gestational age at enrollment in the VDAART discovery or replication cohort were consistent with the effectiveness of the matching procedures used (all $P>0.05$, minimum $P=0.12$; Table 3 ). 250 HD serum levels were significantly lower in women in the VDAART discovery cohort who developed preeclampsia $(P=0.004)$. Overall, in the discovery cohort, 119 subjects had insufficient vitamin D status (<30 ng/ $\mathrm{ml}, 41$ with preeclampsia), and 38 subjects had sufficient vitamin D status ( $\geq 30 \mathrm{ng} / \mathrm{ml}, 6$ positive for preeclampsia), respectively (Figure 3). In the VDAART cohort, 2,829 genes were identified as differentially expressed among women with $25 \mathrm{OHD}$ levels dichotomized at $30 \mathrm{ng} / \mathrm{ml}$ (1,081 upregulated). The pregnant women who developed preeclampsia had 1,547 genes (611 upregulated) that were differentially expressed relative to those of controls. The intersection of the vitamin $\mathrm{D}$ gene signatures and the preeclampsia gene signatures represented vitamin D gene signatures associated with preeclampsia, i.e., 1,323 unique genes (592 upregulated). Of these genes, 348 (158 upregulated) were differentially expressed in peripheral blood in preeclamptic subjects versus controls in the OMEGA study (marginal per gene, $P<0.05$, Figure 3 and Supplemental Table 1; supplemental material available online with this article; doi:10.1172/JCI89031DS1). From the total 348 replicated differentially expressed genes, 33 genes were identified in a database search, and manual curation of the literature revealed an additional 107 genes with a prior known association with preeclampsia (140 of 348, 40.23\%; Supplemental Table 2). The replicated gene signatures also showed tissue-specific enrich- 


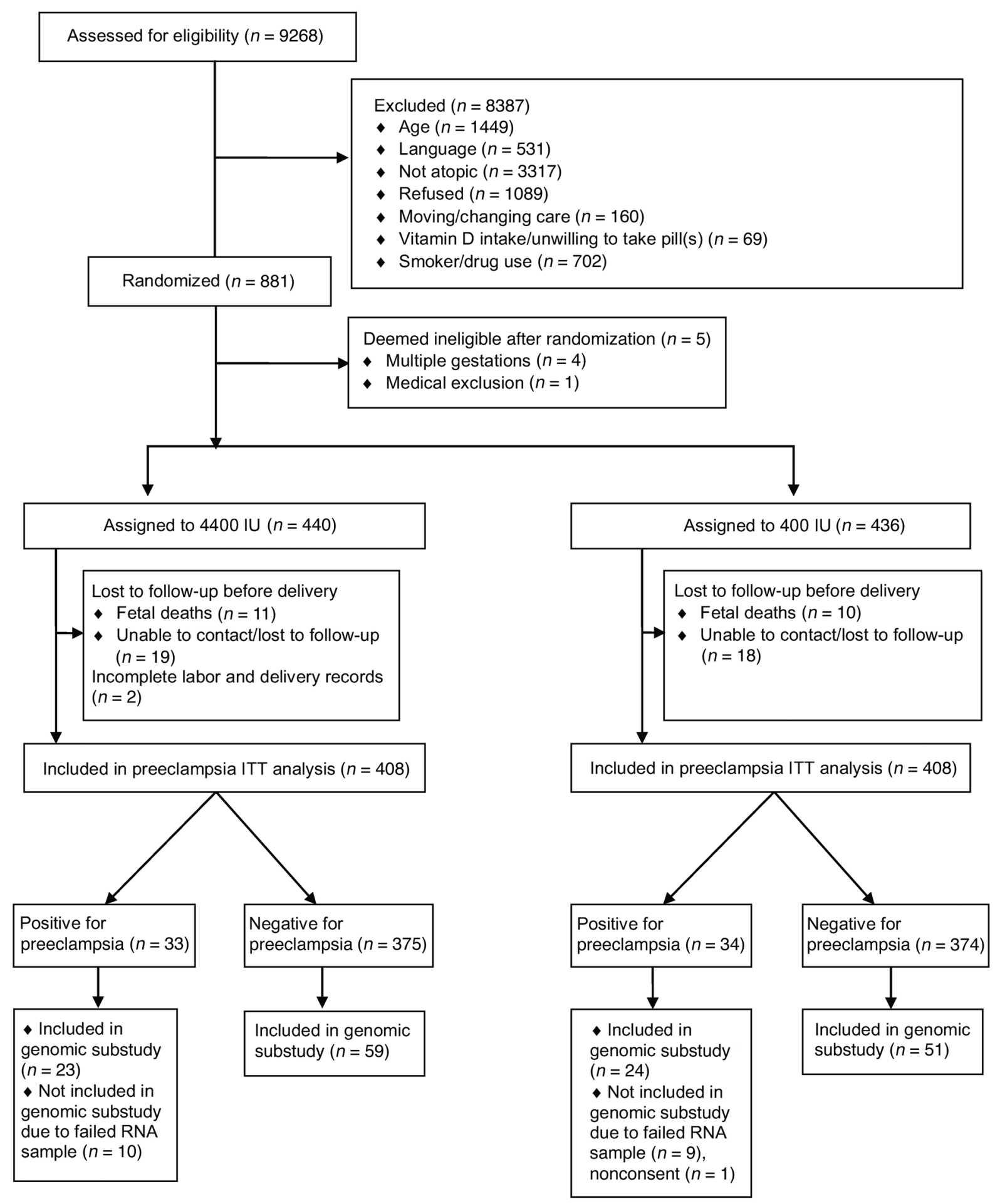

Figure 1. VDAART preeclampsia CONSORT diagram.

ment in placenta $(P<0.0001)$; according to the Human Protein Atlas, 181 genes had evidence of an expression value in placenta (placenta signatures: 181 of 348 [52.01\%], 68 in decidua [19.54\%], 15 in trophoblast [4.31\%], and 98 [28.16] in both; Figure 4 and Supplemental Table 3).

Interactome mapping demonstrates interacting immunomudulatory gene signatures in early pregnancy in women who develop preeclampsia. Three hundred two of three hundred forty-eight
(86.78\%) replicated differentially expressed genes were mapped onto the interactome and demonstrated local clustering in the network (preeclampsia module vs. random expectation, $P<0.0001$; Figure 5). We identified the "observable preeclampsia module" consisting of 143 genes with 552 connections, among them IL8 (also known as CXCL8), MX1, HLA-DP1A, HLA-DBQB1, MMP9, RPL31, ACTA2, IFI44L, and SERPING1, all of which were previously reported to be in association with preeclampsia (Supple- 
Table 1. Baseline maternal characteristics in the VDAART

\begin{tabular}{|c|c|c|c|}
\hline & $4,400 \mathrm{IU}(n=408)$ & $400 \mathrm{IU}(n=408)$ & $P$ value \\
\hline \multicolumn{4}{|l|}{ Clinical center } \\
\hline San Diego, $n(\%)$ & $136(33)$ & $141(35)$ & 0.68 \\
\hline Boston, $n(\%)$ & $118(29)$ & $125(31)$ & \\
\hline St. Louis, $n(\%)$ & $154(38)$ & $142(35)$ & \\
\hline Age in years, mean (SD) & $27.5(5.4)$ & $27.2(5.6)$ & 0.34 \\
\hline$<30, n(\%)$ & $258(63)$ & $268(66)$ & 0.64 \\
\hline $30-34, n(\%)$ & $121(30)$ & $109(27)$ & \\
\hline$\geq 35, n(\%)$ & $29(7)$ & $31(8)$ & \\
\hline Gravidity, including in the VDAART, mean (SD) & $2.6(1.7)$ & $2.3(1.4)$ & 0.008 \\
\hline Cestational age in weeks at enrollment, mean (SD) & $14.2(2.8)$ & $14.2(2.7)$ & 0.86 \\
\hline Gestational age in weeks at delivery, mean (SD) & $38.7(2.13)$ & $38.8(2.42)$ & 0.83 \\
\hline BMl at first appointment, mean (SD) & $28.9(7.7) n=382$ & $29.0(7.6) n=391$ & 0.77 \\
\hline Baseline vitamin D (25OHD) in ng/ml, mean (SD) & $23.32(10.3) n=405$ & $22.6(10.2) n=406$ & 0.29 \\
\hline Third trimester $250 \mathrm{HD}$ in ng/ml, mean (SD) & $39.2(15.34) n=386$ & $26.8(10.74) n=391$ & $<0.0001$ \\
\hline Third trimester $250 \mathrm{HD} \geq 30 \mathrm{ng} / \mathrm{ml}, n(\%)$ & $290(74.2)$ & $135(35)$ & $<0.0001$ \\
\hline \multicolumn{4}{|l|}{ Mother } \\
\hline Asthma, $n(\%)$ & $173(42)$ & $154(38)$ & 0.17 \\
\hline Allergic rhinitis, $n(\%)$ & $258(63)$ & $264(65)$ & 0.66 \\
\hline Eczema, $n(\%)$ & $128(31)$ & $132(32)$ & 0.76 \\
\hline \multicolumn{4}{|l|}{ Race/ethnicity } \\
\hline Black/African-American, $n(\%)$ & $178(44)$ & $177(43)$ & 0.99 \\
\hline White/Hispanic, $n$ (\%) & $55(13)$ & $58(14)$ & \\
\hline White/non-Hispanic, $n(\%)$ & $108(26)$ & $108(26)$ & \\
\hline Other, $n(\%)$ & $67(16)$ & $65(16)$ & \\
\hline \multicolumn{4}{|l|}{ Education level completed } \\
\hline Lower than high school, $n(\%)$ & $63(15)$ & $39(10)$ & 0.052 \\
\hline High school, technical school, $n$ (\%) & $111(27)$ & $133(33)$ & \\
\hline Some college, $n(\%)$ & $98(24)$ & $96(24)$ & \\
\hline College graduate/graduate school, $n(\%)$ & $136(33)$ & $140(34)$ & \\
\hline \multicolumn{4}{|l|}{ Marital status } \\
\hline Married, $n(\%)$ & $179(44)$ & $193(47)$ & 0.59 \\
\hline Divorced/separated, $n(\%)$ & $12(3)$ & $10(2)$ & \\
\hline Not married, $n(\%)$ & $217(53)$ & $205(50)$ & \\
\hline \multicolumn{4}{|l|}{ Household income (US\$) } \\
\hline$<\$ 30,000, n(\%)$ & & $122(30)$ & $117(29)$ \\
\hline$\$ 30,000-49,999, n(\%)$ & $50(12)$ & $55(13)$ & \\
\hline$\$ 50,000-74,999, n(\%)$ & $48(12)$ & $50(12)$ & \\
\hline$\$ 75,000-99,999, n(\%)$ & $42(10)$ & 37 (9) & \\
\hline$\$ 100,000-149,000, n(\%)$ & $33(8)$ & $31(8)$ & \\
\hline$>\$ 150,000, n(\%)$ & $18(4)$ & $13(3)$ & \\
\hline
\end{tabular}

corrected $P<0.05$; Supplemental Table 4). Similarly, the observable preeclampsia module was associated with immune system process, response, and regulation $(N=57,45$, and 27, respectively; all corrected $P<0.05$; Supplemental Table 5).

\section{Discussion}

In the VDAART, supplementation with vitamin $\mathrm{D}$ initiated between 10 and 18 weeks of gestation did not reduce the frequency of preeclampsia in the ITT analysis. However, women who had sufficient vitamin D levels $(\geq 30 \mathrm{ng} / \mathrm{ml})$ at enrollment in early pregnancy and retained that status in late pregnancy had a significantly lower rate of preeclampsia compared with women with insufficient vitamin D levels $(P=0.04)$. This finding, along with the biological significance of the identified peripheral blood gene expression signatures in women who developed preeclampsia, implicates early pregnancy vitamin $\mathrm{D}$ status in the development of the disease.

Despite evidence from observational studies showing an association of low vitamin D status during pregnancy with preeclampsia (3-6), few randomized trials have examined the effectiveness of vitamin D supplementation in pregnancy outcomes, particularly in preventing preeclampsia $(5,7,8,18,19)$. These trials had a smaller sample size, and in only 1 study was the therapy initiated approximately as early as it was in the VDAART

mental Table 2) and showed the highest degree of connectivity in the interactome. This module was significantly close to known signaling pathways for both IL-10 and vitamin D ( $Z$ scores -8.6 and -3.8, respectively, $P \leq 0.0001$; Figure 6 and Table 4). Gene ontology (GO) enrichment analysis linked the 348 replicated gene signatures to several immunologic functions including immune system process, response, and regulation $(N=109,83$, and 63, respectively); innate and adaptive immune response $(N=50$ and 19 , respectively); inflammatory response $(N=35)$; IL-10 production and negative regulation ( $N=8$ and 7, respectively); regulation of cytokine production $(N=25)$; and type I IFN-signaling pathway and cellular response to type I IFN $(N=10$ and 10 , respectively, all
(8). Except for the 1 other trial started early in pregnancy (8) and a recent trial of vitamin D supplementation after 20 weeks of gestation (19), the doses of vitamin D used were less than those in VDAART. No other trial, to our knowledge, has explored the underlying biological mechanisms using expression profiling and functional annotation of identified genes.

The inverse association between maternal vitamin D concentrations in early pregnancy and risk of preeclampsia in the VDAART is consistent with the findings of previous observational studies (20-22). A noteworthy finding of the VDAART is that a circulating vitamin $\mathrm{D}$ level of approximately $40 \mathrm{ng} / \mathrm{ml}$ during pregnancy seems to minimize the vitamin $\mathrm{D}$-associated risk of 


\section{Table 2. Logistic regression; effects of treatment arm and vitamin D levels on preeclampsia}

\begin{tabular}{|c|c|c|c|}
\hline Terms & $\begin{array}{l}\text { Univariate } \\
\text { OR ( } 95 \% \mathrm{Cl})\end{array}$ & $\begin{array}{c}\text { Multivariable (all covariates) } \\
\text { OR ( } 95 \% \mathrm{Cl})\end{array}$ & $\begin{array}{l}\text { Stepwise regression } \\
\text { adjusted OR (95\% Cl) }\end{array}$ \\
\hline \multicolumn{4}{|l|}{ Treatment arm } \\
\hline Active & 1 & 1 & - \\
\hline Placebo & $1.03(0.62-1.70)$ & $0.7(0.4-1.29)$ & - \\
\hline \multicolumn{4}{|l|}{ First and third trimester vitamin D } \\
\hline Low-low & 1 & 1 & 1 \\
\hline Low-high or high-low & $0.62(0.37-1.04)$ & $0.68(0.37-1.28)$ & $0.79(0.45-1.38)$ \\
\hline High-high & $0.19(0.06-0.6)$ & $0.25(0.07-0.87)$ & $0.28(0.08-0.96)^{A}$ \\
\hline \multicolumn{4}{|l|}{ Maternal age (yr) } \\
\hline$<35$ & 1 & 1 & - \\
\hline$\geq 35$ & $0.78(0.27-2.24)$ & $0.96(0.27-3.3)$ & - \\
\hline $\begin{array}{l}\text { Maternal BMl at first appointment } \\
\left(\mathrm{kg} / \mathrm{m}^{2}\right)\end{array}$ & $1.05(1.02-1.08)$ & $1.04(1.002-1.07)$ & $1.04(1.01-1.07)^{\mathrm{B}}$ \\
\hline \multicolumn{4}{|l|}{ Maternal marital status } \\
\hline Not married/divorced & 1 & 1 & - \\
\hline Married & $0.52(0.3-0.88)$ & $0.7(0.33-1.60)$ & \\
\hline \multicolumn{4}{|l|}{ Annual family income } \\
\hline$<\$ 50,000$ & 1 & 1 & - \\
\hline$\geq \$ 50,000$ & $0.75(0.4-1.4)$ & $1.4(0.77-2.5)$ & - \\
\hline Unknown or refused to report & $1.4(0.6-3.4)$ & $1.36(0.7-2.6)$ & - \\
\hline \multicolumn{4}{|l|}{ Gravidity } \\
\hline Multigravid & 1 & 1 & 1 \\
\hline Nulligravid & $1.37(0.82-2.27)$ & $1.7(0.9-3.0)$ & $1.70(1.003-3.03)$ \\
\hline \multicolumn{4}{|l|}{ Clinical centers } \\
\hline Site 1 & 1 & 1 & 1 \\
\hline Site 2 & $1.01(0.49-2.06)$ & $0.60(0.22-1.5)$ & $0.69(0.32-1.49)$ \\
\hline Site 3 & $2.05(1.12-3.7)$ & $0.99(0.39-2.5)$ & $1.52(0.79-2.92)$ \\
\hline \multicolumn{4}{|l|}{ Race/ethnicity } \\
\hline White/mixed European descent & 1 & 1 & - \\
\hline Black/African-American & $1.65(0.94-2.9)$ & $1.07(0.47-2.4)$ & - \\
\hline Other & $1.2(0.55-2.6)$ & $0.95(0.37-2.4)$ & - \\
\hline \multicolumn{4}{|l|}{ Maternal education level } \\
\hline Lower than college & 1 & 1 & 1 \\
\hline College and above & $0.36(0.18-0.7)$ & $0.43(0.17-1.08)$ & $0.4(0.21-0.84)^{c}$ \\
\hline \multicolumn{4}{|c|}{$\begin{array}{l}\text { Categories of } 250 \mathrm{HD} \text { levels: high-high, subjects who had } \geq 30 \mathrm{ng} / \mathrm{ml} \text { at randomization and in the third } \\
\text { trimester; high-low, subjects who had } \geq 30 \mathrm{ng} / \mathrm{ml} \text { at randomization but }<30 \mathrm{ng} / \mathrm{ml} \text { in the third trimester; low- } \\
\text { high, subjects who had }<30 \mathrm{ng} / \mathrm{ml} \text { at randomization but } \geq 30 \mathrm{ng} / \mathrm{ml} \text { in the third trimester; low-low, subjects } \\
\text { who had }<30 \mathrm{ng} / \mathrm{ml} \text { at both time points. }{ }^{A} P=0.04,{ }^{B} P=0.016 \text {, and }{ }^{C} P=0.015 \text {. }\end{array}$} \\
\hline
\end{tabular}

vitamin $\mathrm{D}_{3}$ supplementation (24). These observations suggest that the current criterion for vitamin D deficiency $(<20 \mathrm{ng} / \mathrm{ml})$ of the Institute of Medicine (IOM) may be a suboptimal vitamin D level for pregnancy-related adverse events such as preeclampsia. Our observation is also consistent with the contemporary understanding of preeclampsia as arising from a preclinical phase of aberrant placentation in early pregnancy (25). These data suggest that earlier supplementation with vitamin $\mathrm{D}$, even prior to implantation of the embryo, may be needed to improve vitamin D status in pregnant women to prevent preeclampsia, a notion that should be examined in a clinical trial.

Our gene expression substudy identified a set of vitamin D-associated genes related to preeclampsia, and network-based closeness demonstrated their connectivity to known vitamin $\mathrm{D}$-signaling pathways, indicating the functional cohesiveness of vitamin D to the preeclampsia disease module. Most of the genes in the replicated module were associated with maternal systemic changes in immune (innate and humoral) and inflammatory responses. Among the functional interactions, one of the identified biological processes is negative regulation and production of IL-10 expression. IL-10 is an antiinflammatory cytokine that also mediates innate and humoral immune responses. Decreased

preeclampsia. Our data are consistent with the NICHD trial's conclusion that a level of $40 \mathrm{ng} / \mathrm{ml}$ of circulating $25 \mathrm{OHD}$ is required to support maximum $1,25(\mathrm{OH})_{2} \mathrm{D}$ production during pregnancy $(8$, 23). In the NICHD trial, 4,000 IU versus 2,000 and 400 IU vitamin D were given after 12 to 16 weeks of gestation, with 4,000 IU having the greatest effect on reducing the composite rate of pregnancy comorbidities. Furthermore, across the treatment arms of this trial (400, 2,000, and 4,000 IU vitamin D), there was a trend toward a significant effect on the odds of pregnancy hypertensive disorder including preeclampsia after controlling for race $(P=$ 0.05) (8). The observed effect is consistent with the findings of a recent trial of vitamin D supplementation (60,000-480,000 IU dose determined by a baseline 25OHD of $>20,10-20$, and $<10$ $\mathrm{ng} / \mathrm{ml} ; 49 \%<10 \mathrm{ng} / \mathrm{ml}$ ) after 20 weeks of gestation (19). As with the NICHD trial, we did not observe any adverse events due to
IL-10 levels in placenta and in serum of women with preeclampsia have been observed $(26,27) .1,25(\mathrm{OH})_{2} \mathrm{D}_{3}$ decreases the synthesis of IL-12 and IL-23 and increases the production of IL-10 by DCs (28). This effect results in a decrease in Th1 cell responses, producing more Tregs and a Th2 profile that favors a state of immune tolerance to the fetus in the mother (29). IL-10 has also been implicated in placental angiogenesis, growth, and remodeling and in blunting hypoxic injury at the maternal-fetal interface $(29,30)$. Additionally, the identified preeclampsia module of replicated genes was significantly close and connected to the known IL-10 pathways in the interactome $(P \leq 0.0001)$.

Why was therapy with vitamin D not successful in modifying the incidence of preeclampsia in the VDAART or in previous studies? It is possible that vitamin D supplementation and the subsequently increased maternal vitamin D metabolites are not import- 


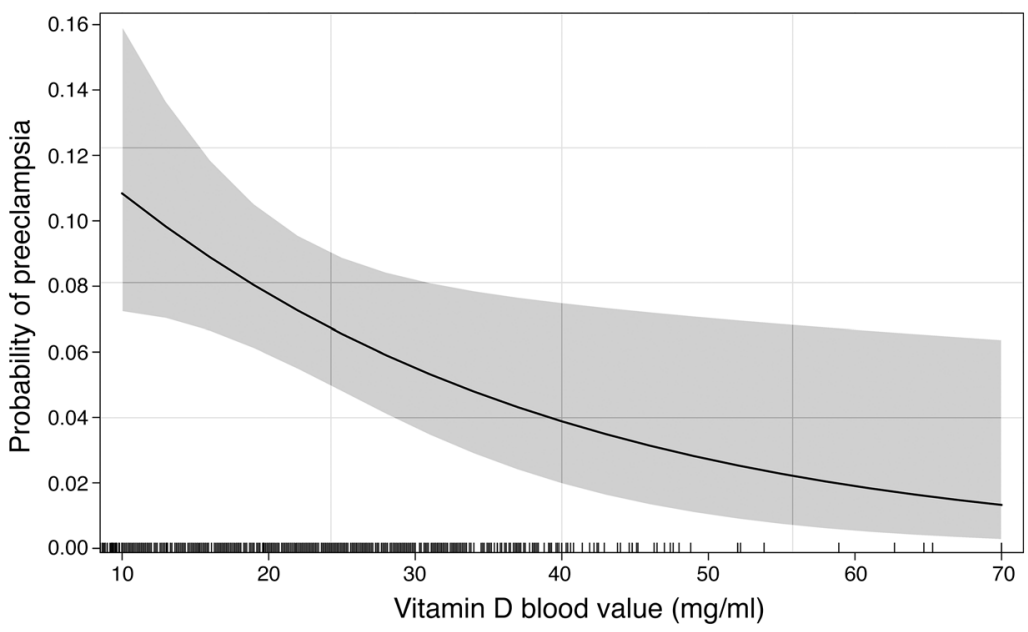

Figure 2. Dose-response association between maternal serum 250HD concentrations at 10 to 18 weeks of gestation and the corresponding predicted probability of preeclampsia derived from a logistic regression model adjusted for the identified confounders (Table 2). The gray zone indicates 0.95 confidence bands $\left(N_{\text {preeclampsia }}=67, N_{\text {healthy }}\right.$ $=749$ ). The minimum predicted risk for the population under study relative to the distribution of pregnant women (vertical tick marks on the $x$ axis) falls at the concentrations of 40 to $50 \mathrm{ng} / \mathrm{ml}$ serum vitamin $\mathrm{D}$. ant in the modification of pathophysiologic alterations involved in the development of preeclampsia. However, the fact that the observed association of maternal vitamin D levels with a risk of preeclampsia was reported in several other studies under different study conditions $(5,8)$ leads us to deduce that the observed association in the VDAART results is worthy of further investigation, and that conclusion is supported by our gene expression study.

The timing of vitamin D administration and achievement of optimal vitamin D concentration are important. Immunologic evidence, detection of early biomarkers, as well as aberrant placental development and function suggest that preeclampsia is a disorder of early placentation $(28,31-35)$. Sufficient vitamin D levels need to be present at the time of implantation and placentation, the 2 stages in which maternal-fetal immune interfaces play an important role in preeclampsia $(36,37)$. A significant effect of vitamin $\mathrm{D}_{3}$ on human extravillous trophoblast invasion in early pregnancy was observed at a concentration of $40 \mathrm{ng} / \mathrm{ml}$ (38). Our gene expression substudy showed the existence of distinctive biological processes consistent with preeclampsia as early as the tenth week of pregnancy in women with low vitamin D status who went on to develop preeclampsia compared with what was observed in the vitamin D-sufficient matched controls. These observations are suggestive of the evolution of systematic immunologic alterations at the earliest placental stage and prior to the time of enrollment in the VDAART. On the contrary, those with vitamin D sufficiency at trial entry $(21.8 \%)$ and who maintained that status through their late pregnancy demonstrated a considerably lower risk of preeclampsia. Although vitamin D is implicated in the pathophysiology of preeclampsia, it is possible that the role of vitamin D is mediated by other interacting factors (environmental or genetic), such that a subgroup of pregnant women with low vitamin D status might achieve the greatest benefit from this therapy. The observed variability in a dose-response association between maternal vitamin D levels and risk of preeclampsia, particularly at lower concentrations, supports this notion (Figure 2). It should also be noted that the 4,000 IU dose of vitamin $\mathrm{D}_{3}$ in the VDAART resulted in a sufficient level of serum vitamin D ( $\geq 30 \mathrm{ng} / \mathrm{ml}$ ) in only $74 \%$ of the pregnancies at 32 to 38 weeks of gestation, which is less than the $82 \%$ at 1 month prior to delivery in the NICHD trial on a similar dose (23). The results of these 2 studies highlight the latest emerging evidence that higher supplementation doses (2,000-6,000 IU/day) than the current recommendation of 400 to $600 \mathrm{IU} /$ day, particularly in the absence of adequate sun exposure or other highrisk conditions of vitamin D deficiency, might be required to support sufficient levels throughout pregnancy. While the current evidence from randomized controlled trials (RCTs) is not sufficient to support 25OHD screening and high-dose vitamin $\mathrm{D}$ supplementation (>2,000 IU) in all pregnant women $(39,40)$, screening and modification of vitamin $\mathrm{D}$ status in early pregnancy and ideally before pregnancy for women with a high risk of vitamin D deficiency should be considered. Furthermore, for to-be pregnant women with a high risk of vitamin $\mathrm{D}$ deficiency as well as a risk of nonadherence to vitamin D sup- 


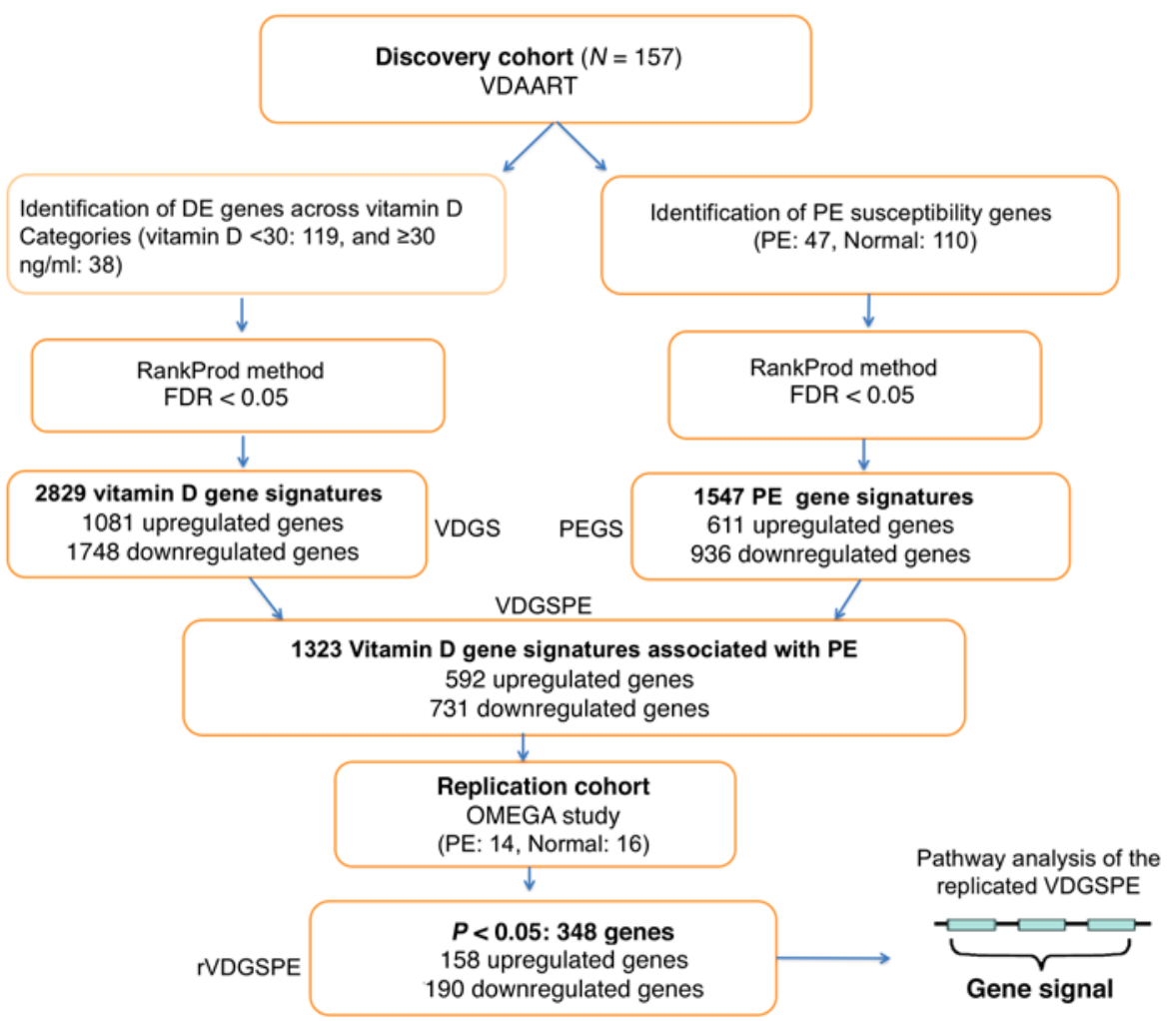

Figure 3. Gene expression study flow chart. Summary of analysis approach and findings on gene expression analysis, through discovery and replication stages. Gene expression study was conducted using RankPord to identify differentially expressed genes in both discovery and replication cohorts ( $N=157$ and 30 , respectively). $\mathrm{PE}$, preeclampsia; $\mathrm{DE}$, differentially expressed; VDCS, vitamin D gene signatures; PECS, preeclampsia gene signatures; VDCSPE, vitamin $\mathrm{D}$ gene signatures associated with preeclampsia; rVDCSPE, replicated vitamin D gene signatures associated with preeclampsia; $\mathrm{PPI}$, protein-protein interaction. plementation, prepregnancy supplementation strategies such as a high dose of vitamin $\mathrm{D}_{3}$ in addition to individualized supplementation doses should be considered, and frequent 25OHD measurements during pregnancy are recommended (41). These concepts need to be definitively tested in a prepregnancy clinical trial in a population of women at high risk of preeclampsia.

A limitation of the VDAART as well as other RCTs of vitamin D supplementation during pregnancy was that subjects with vitamin D sufficiency, i.e., those with a level above $30 \mathrm{ng} / \mathrm{ml}$, were not screened and were excluded from the trial. While this approach might have affected the efficacy of vitamin D supplementation, it provided the opportunity to obtain valuable knowledge by allowing us to compare the pregnant women with vitamin D sufficiency with those with vitamin D insufficiency in terms of their risk of preeclampsia occurrence and to identify potential mechanisms of preeclampsia in early pregnancy. It is noteworthy that a maternal or paternal history of allergic diseases has not been recognized as a risk factor for preeclampsia; nevertheless, this inclusion criterion for the VDAART might limit the broad generalizability of our results in certain populations of pregnant women.

In conclusion, 4,400 IU daily supplementation of vitamin D initiated at 10 to 18 weeks of gestation did not show a protective effect against preeclampsia in our ITT analysis; however, vitamin D sufficiency $(\geq 30 \mathrm{ng} / \mathrm{ml}$ ) at randomization and at 32 to 38 weeks of gestation was associated with a significant $(P=0.04)$ effect on reducing the risk of preeclampsia development. This observation and the identified peripheral blood gene expression patterns of preeclamptic women showing immune gene activation at 10 to 18 weeks of gestation highlight the importance of vitamin D sufficiency in early pregnancy.

\section{Methods}

VDAART design, participants, interventions, and oversight. The VDAART was a randomized, double-blind, placebo-controlled clinical trial of vitamin D supplementation (4,000 IU vitamin D plus a multivitamin with 400 IU vitamin D daily) versus placebo (placebo pill plus a multivitamin with 400 IU vitamin D daily) for pregnant women with a high risk of atopic disease in their family; the goal of the trial was to prevent asthma in children as well as predefined pregnancy outcomes, including preeclampsia. Participants were $10-18$ weeks pregnant and between the ages of 18 and 40 years when recruited at obstetric clinics at 3 centers in the United States: Boston University Medical Center in Boston, Massachusetts; Washington University in St. Louis, Missouri; and Kaiser Permanente Southern California Region in San Diego, California.

Eligible participants were screened between October 2009 and July 2011 and enrolled if they met the eligibility criteria. Compliance with daily pill taking during pregnancy was monitored via the Medical Event Monitoring System (MEMS) (www.aardexgroup.com). The Data and Safety Monitoring Board (DSMB), convened by the National Heart, Lung, and Blood Institute (NHLBI), met every 6 months to review recruitment, adherence, adverse events, data quality, and protocol changes. Details of the trial design and protocol as well as further information on the screening, random allocation and blinding process, compliance assessment, statistical power, and trial monitoring have been published previously $(24,42)$. The results of the trial were prepared and reported in accordance with the Consolidated Standards of Reporting Trials (CONSORT) guidelines.

VDAART and eligibility. The VDAART (www.vdaart.com) was sponsored by the NHLBI and registered at ClinicalTrials.gov (NCT00920621). The VDAART involved pregnant women with singleton pregnancies at 10 to 18 weeks of gestation, who were enrolled in 


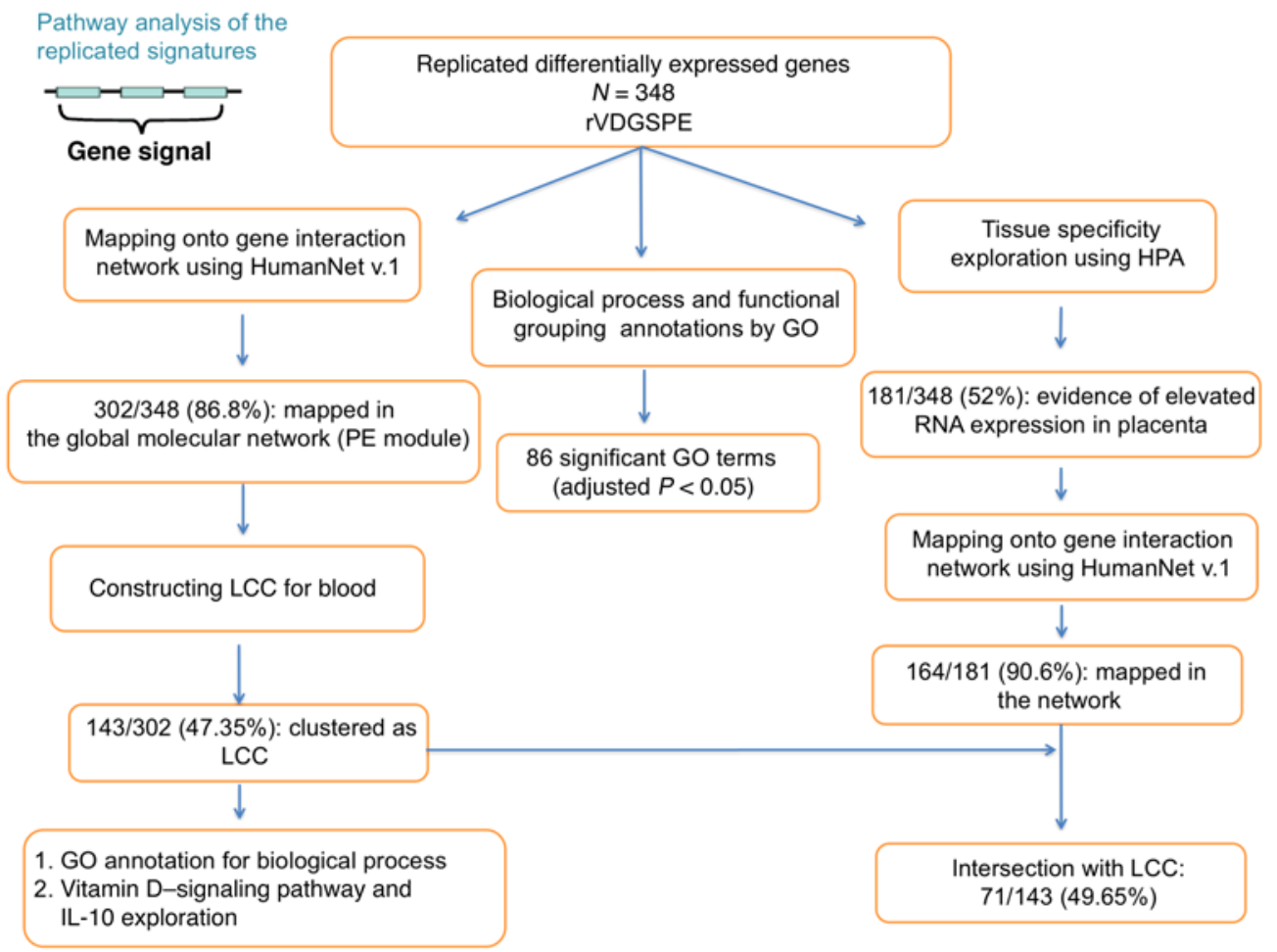

Figure 4. Functional annotation, tissue specificity, and PPI network mapping of vitamin D signatures associated with preeclampsia obtained from the gene expression study. GO functional enrichment analysis was based on a hypergeometric test with an FDR adjustment for multiple testing of the identified pathways. a multicenter, randomized trial for assessment of the defined outcome of pregnancy after receiving either vitamin D (cholecalciferol, 4,000 $\mathrm{IU} /$ day, equivalent to $100 \mu \mathrm{g} /$ day) or placebo. All pregnant participants received prenatal vitamins containing $400 \mathrm{IU}(10 \mu \mathrm{g} /$ day) of cholecalciferol; thus, the vitamin D treatment arm received a total of 4,400 IU/ day $(110 \mu \mathrm{g} /$ day $)$, and the placebo arm received $400 \mathrm{IU} /$ day $(10 \mu \mathrm{g} /$ day). The pregnant women's clinical course was followed prospectively from randomization (10-18 weeks) to the end of the pregnancy and for investigation of predefined pregnancy outcomes including preeclampsia, preterm birth, and term delivery. The inclusion criteria were as follows: (a) Maternal history or biological paternal history of asthma, eczema, or allergic rhinitis. Pregnant women were considered asthmatic or allergic if they gave a positive answer to the direct question in the enrollment questionnaire or any subsequent monthly questionnaire; (b) gestational age between 10 and 18 weeks at the time of randomization; (c) maternal age between 18 and 39 years; (d) not a current smoker (defined as not having smoked for at least 1 month prior to enrollment) and not a user of other nicotine products (e.g., nicotine patch) for at least 1 month prior to enrollment; (e) English- or Spanish-speaking; (f) intention to participate in the trial throughout the pregnancy.

The exclusion criteria were as follows: (a) Gestational age beyond 18 weeks; (b) presence of any of the following chronic medical conditions: (i) hypertension (on medication); (ii) diabetes mellitus; (iii) parathyroid disease; (iv) uncontrolled thyroid disease; (vi) kidney stones; or (vii) sarcoidosis; (c) intake of vitamin D supplements containing more than 2,000 IU/day of vitamin D3; (d) multiple gestational pregnancy; (e) pregnancy achieved by assisted reproductive techniques (e.g., IUI, IVF); (f) current use of illicit drugs (defined as any use in the past 6 months prior to enrollment); (g) previous enrollment in the VDAART for a prior pregnancy; (h) any major fetal anomalies detected prior to delivery; (i) patient health questionnaire (PHQ-9) depression scale of 15 or higher; (j) any condition, in the opinion of the clinical center's principal investigator, that would inhibit compliance with the study medications or prohibit long-term participation in the trial.

Assessment of compliance: MEMS. The primary measure of compliance with pill taking was monitored via the MEMS (AARDEX Group; http://www.aardexgroup.com), which consists of a pill bottle equipped with special cap that includes an electronic microchip that stores the date and time of each opening of the bottle. Each monitor recorded the time and date of each opening and closing of the container through integrated microcircuitry. The MEMS stores up to 3,800 medication events in nonvolatile, electrically erasable, programmable read-only memory (EEPROM), allows wireless data transfer, fits standard pharmacy bottles, provides 36 months of battery life, and is water resistant and CE marked. Monitors were designed for usage by 1 patient with 1 drug. A reader allowed transfer of the dose-timing data from the MEMS to a Microsoft Windows-based computer. Data from the MEMS were downloaded at the monthly in-person prenatal visits and after delivery. The data from the prenatal follow-up visits were used to provide feedback to the participant, reinforcing those participants with good adherence and providing extra teaching and encouragement for those with suboptimal adherence. The data from both prenatal follow-up and postnatal downloads were used to derive a summary adherence measure, to be used as a covariate in our analyses. In addition to the information obtained from the MEMS, the 25OHD levels detected from the baseline measurement and at the third-trimester visits provided excellent adherence information for this trial.

Data collection on the VDAART subjects. The research staff met with the participants monthly, in conjunction with their regularly scheduled obstetrical visits. At these visits, a short maternal health questionnaire was administered, MEMS cap information was downloaded, and study medication and prenatal vitamins were refilled. At these monthly prenatal visits, the research staff conducted monthly reviews of electronic medical records to check for pregnancy compli- 


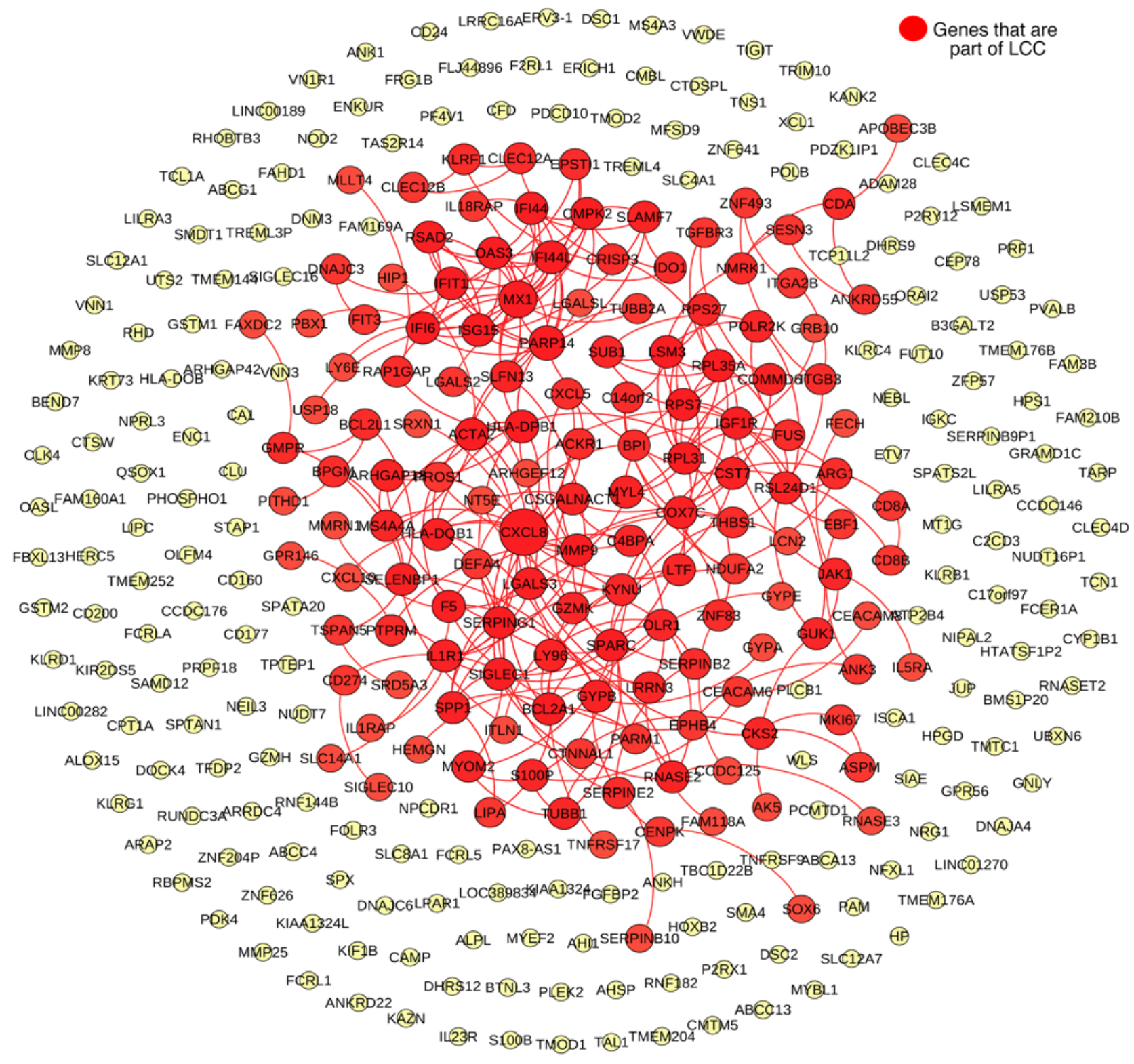

\section{Term}

Immune system process

Immune response

Defense response

Response to stress

Response to biotic stimulus

Regulation of immune system process

Innate immune response

Inflammatory response

Platelet degranulation

Immune effector process

Cellular response to cytokine stimulus

Leukocyte migration

Negative regulation of immune system proces

Cytokine-mediated-signaling pathway

Cell-surface receptor-signaling pathway

$\mathrm{IL}-10$ production

Cellular response to type I IFN

Type I IFN-signaling pathway

Response to type I IFN

Regulation of cell migration

Cytokine production

Lymphocyte-mediated immunity

Adaptive immune response

Regulation of cytokine production

$\begin{array}{cl}\begin{array}{c}\text { No. of involved } \\ \text { genes }\end{array} & \begin{array}{c}\text { Corrected } \\ \boldsymbol{P} \text { value }\end{array} \\ 109 & 1.31 \times 10^{-16} \\ 83 & 1.12 \times 10^{-15} \\ 82 & 4.07 \times 10^{-13} \\ 125 & 1.28 \times 10^{-9} \\ 50 & 5.27 \times 10^{-9} \\ 63 & 1.17 \times 10^{-6} \\ 50 & 0.000001 \\ 35 & 0.0000042 \\ 12 & 0.0000606 \\ 36 & 0.000174 \\ 34 & 0.000536 \\ 22 & 0.000583 \\ 22 & 0.00102 \\ 29 & 0.00139 \\ 81 & 0.00143 \\ 8 & 0.00157 \\ 10 & 0.00294 \\ 10 & 0.00294 \\ 10 & 0.00411 \\ 29 & 0.00733 \\ 28 & 0.0127 \\ 15 & 0.0364 \\ 19 & 0.043 \\ 25 & 0.0498\end{array}$

Figure 5. LCC genes (143 genes, highlighted in red) corresponding to the "observable preeclampsia module" among the replicated differentially expressed genes $(\boldsymbol{N}=\mathbf{3 4 8})$ and their biological processes. The connectivity of the replicated signatures was explored by mapping onto functional protein-protein interaction networks from HumanNet v.1. 


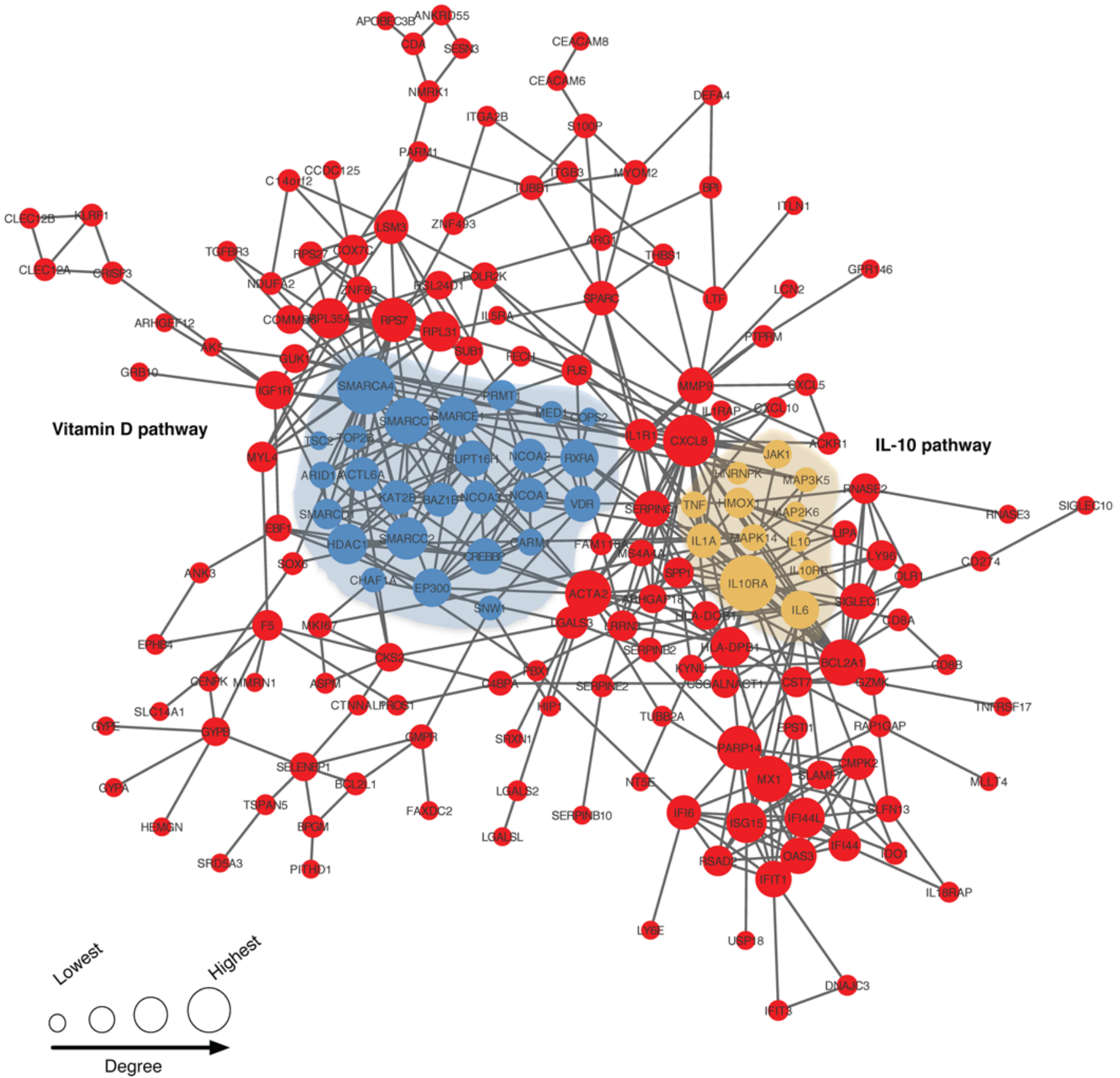

Figure 6. Connection among the vitamin D- and IL-10-signaling pathway genes (blue and yellow modules, respectively) within the module. Only the direct connections between vitamin $D$ and IL-10 pathway genes within the module are shown. The size of the circles corresponds to the degree of genes in the interactome. The closeness of the vitamin D- and IL-10-signaling pathways to the LCC is based on the observed shortest path between each set of genes and the LCC.

cations; these were logged, and a severe adverse event (SVE) form or an adverse event form was completed as applicable. At 32 to 38 weeks of gestation, in addition to the monthly routine, a blood draw, skin pigmentation determination, and several of the questionnaires that were administered at the enrollment visit were repeated. At delivery, cord blood was collected, and the research staff collected information regarding the type of delivery, birth weight of the baby, and other anthropometric measures from the delivery records.

Study outcome and main predictor measurements. The occurrence of preeclampsia was a prespecified secondary outcome of the trial and an adverse event. Pregnant women with known chronic hypertension were excluded from entry into the VDAART. Every month, study coordinators performed a medical record review for cases of preeclampsia as part of the protocol for identifying SVEs in the trial. Medical records were abstracted for all participants after delivery. A committee of 4 board-certified obstetricians conducted a blinded review of 276 abstracted charts of subjects with a noted diagnosis of hypertension, proteinuria, or preeclampsia to determine preeclampsia status. The diagnosis of preeclampsia at the time of the record reviews was based on the American Congress of Obstetricians and Gynecologists (ACOG) 
Table 4. Average distances between the preeclampsia module and vitamin D receptor (VDR) and IL-10 pathways

\section{Pathway closeness to PE module}

IL-10 pathway vs. preeclampsia module

VDR pathway vs. preeclampsiamodule

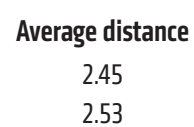

2.45

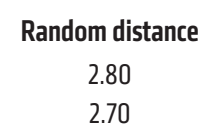

2.80

2.70

\section{SD \\ 0.04 \\ 0.045}

\begin{abstract}
Z score
$-8.60$

$-3.80$
\end{abstract}

guidelines (43), which included the identification of high blood pressure (BP) and either proteinuria ( $\geq 300 \mathrm{mg}$ per 24 -hour collection or $\geq 1+$ on a urine dipstick) or the presence of elevated liver enzymes, high platelet count, headache, or visual disturbances after 20 weeks of gestation. High BP was diagnosed for participants who had a systolic BP of $140 \mathrm{mmHg}$ or higher, a diastolic $\mathrm{BP}$ of $90 \mathrm{mmHg}$ or higher, or both, with a second elevated measurement noted in the medical record at least 6 hours after the first measurement was taken. Consequently, preeclampsia was diagnosed in 67 participants. Total 25OHD levels were measured using a chemiluminescence assay on a DiaSorin LIAISON machine at the data coordination center (DCC). We performed the 25OHD TOTAL assay, which has shown an excellent correlation with the DiaSorin 25OHD RIA assay, which has been used to defined reference levels. This method has also exhibited better concordance with liquid chromatography-tandem mass spectrometry (LC-MS/MS) results as compared with other available methods (44). Additionally, using specific polyclonal antibodies, this method avoids the previously observed racial differences in vitamin D-binding protein (VDBP) concentrations that are most likely due to monoclonal assay bias $(45,46)$. For quality control, our laboratory used the US National Institute of Standards and Technology (NIST) level 1 protocol.

Gene expression substudy. Participants of the preeclampsia microarray study comprised a nested case-control group $(N=157)$ selected from among participants of the VDAART. Of the 67 women with preeclampsia, 47 (70.14\%) had a suitable RNA sample available at entry into the trial (10-18 weeks of gestation) and participated in the gene expression substudy. Controls were chosen from the VDAART subjects matched for age (within 5 years), race, and study center, such that each subject had 2 matched controls. Once the initial set of matched study subjects and controls was formed, additional controls were incorporated to enhance the range of 25OHD concentrations present among the control subjects (total $N_{\text {controls }}=110$ ). In a post-matching comparison, we found no significant difference (all $P$ values $>0.05$ ) in age, race, or maternal gestational age in early pregnancy among the 47 preeclampsia subjects and the 110 normal controls.

RNA isolation and microarray processing. Total RNA was isolated from whole blood using the QIAGEN PAXgene Blood RNA Kit according to the manufacturer's protocol. The GLOBINclear Kit (Ambion) was used to remove $\alpha$ and $\beta$ globin mRNA from the sample to increase the sensitivity of the gene expression assays by improving the detection rate of expressed genes. The RNA was quantified using the Nanodrop 8000 and checked for high integrity before preparation of cDNA. The integrity of RNA samples was assessed using the Agilent 2100 Bioanalyzer, and purity of the samples was confirmed using the NanoDrop spectrophotometer and RNA integrity number (RIN $\geq 8$ ). Gene expression was assessed using the Affymetrix Human Gene 1.0 ST Array. Biotinylat- ed cRNA was prepared according to the manufacturer's protocol, and hybridization was processed according to the protocol for the GeneChip Hybridization Control Kit (http://www.affymetrix.com/ estore/browse/products.jsp;jsessionid=7F717F80BE253ABBF6155B16AC95C6F9 ? avMode $=34000 \&$ product $I=131453 \&$ navAction $=$ jump\&aId=productsNav\#1_1).

After image production and processing, an expression set with 33,297 probes and 157 samples, collected at 10 to 18 weeks of gestation (enrollment), were generated, representing the 47 subjects with preeclampsia and the 110 unaffected normal pregnant women. In order to check the quality of the arrays, quantiles of expressions were examined before and after normalization. Background adjustment, $\log _{2}$ transformation, and quantile normalization were performed using the robust multiarray analysis ("rma") function in R BioConductor's "affy" library to minimize nonbiological variability among arrays. Principal component (PC) analysis of samples was done to identify any extreme outlier. Thereafter, phenotypes of interest were merged with the expression set. Probes were annotated using the annotation package for Bioconductor pd.hugene.1.0.st.v1 and confined to annotated probes of autosomal chromosomes (19,137 probes). The interquartile range (IQR) filter (values of IQR including $80 \%$ of the probe expressions) was implemented in $\mathrm{R}$ using the "genefilter" package from Bioconductor to remove expressions showing little changes within arrays (47). This process resulted in an expression set of 15,309 probes belonging to 157 samples collected at 10 to 18 weeks of pregnancy, representing 47 preeclampsia cases and 110 women. To capture the expression heterogeneity, surrogate variable analysis was conducted by applying the surrogate variable analysis (SVA) function to the expression data. Accordingly, 16 surrogate variables (SVs) were identified and expression data adjusted for 12 batches uncorrelated to the main predictor of interest, i.e., vitamin D serum levels in early pregnancy. Thereafter, differential expression analysis was carried out using the Bioconductor package "RankProd," which implements the rank product method for identifying differentially expressed genes (48).

The data discussed in this article were deposited in the NCBI's Gene Expression Omnibus (GEO) database (GEO GSE85307; http://www.ncbi.nlm.nih.gov/geo/query/acc.cgi?token=epajoakqppkvbub\&acc=GSE85307).

Microarray validation by quantitative reverse transcription PCR. To ensure internal validity, the top ranked up- or downregulated genes by RankProd from the replication results were chosen for validation by quantitative reverse transcription PCR (qRT-PCR), and qRT-PCR was performed.

Replication cohort. A population selected to replicate the gene expression results in the VDAART was obtained from a nested case-control study of preeclampsia $\left(N_{\text {cases }}=16\right.$ and $\left.N_{\text {controls }}=16\right)$ with- 
in the OMEGA study, a prospective cohort study designed to assess dietary and other lifestyle risk factors of preeclampsia and other medical complications of pregnancy (49). Participants were nulliparous women, who initiated prenatal care at the Swedish Medical Center in Seattle, Washington, USA. Transcriptional data for 2 cases were of low quality and therefore omitted from the analysis.

Quality control of the replication data. Gene expression of subjects was profiled using Affymetrix GeneChip Arrays. Quantiles of raw expression and principal compontents (PCs) across arrays were examined before and after background adjustment normalization and $\log _{2}$ using the "rma" function in the R "affy" library. Expression data for 2 of the samples were identified as outliers and removed, and quantile-normalized and background-adjusted expression data as well as PCs were reexamined. The results were compared by running the "QCReport" function in the R library's "affyQCReport." The microarray expression data were annotated using "hgu133plus2," available on Bioconductor. The annotated expression from autosomal chromosomes was included in the analysis. The final expression data set contained 40,003 rows of background-adjusted, $\log _{2}$-transformed, rma-summarized, and quantile-normalized expression data for 14 preeclampsia cases and 16 controls.

Statistics. In accordance with the ITT paradigm, data for all women $(N=816)$ were analyzed according to the group to which they were randomly assigned (Figure 1). For demographic data, continuous variables were compared using the appropriate 2-tailed $t$ test or $\chi^{2}$ test, and a $P$ value of less than 0.05 was considered statistically significant.

A vitamin D level of $30 \mathrm{ng} / \mathrm{ml}(75 \mathrm{nmol} / \mathrm{l})$ was used to differentiate between high and low levels (high-high, high-low or low-high, and low-low) at both study time points. Selection of this cutoff was mostly based on the observations of several studies designed to investigate the relationship between serum parathyroid hormone (PTH) and 25OHD levels in adults. These studies have shown that the suppression of PTH reaches a plateau when the $25 \mathrm{OHD}$ level approximates $30 \mathrm{ng} / \mathrm{ml}$ (50). Of note, it has been demonstrated that during pregnancy, serum PTH levels are not different from those of nonpregnant women $(51,52)$.

Two ITT analyses were performed: (a) For the primary outcome assessment, we used logistic regression analysis, controlling for a priori covariates or potential confounders (associated with primary outcome and the main variable of vitamin D status at a significance level of 0.2; Table 2 and Supplemental Table 6) including gravidity, age, race, BMI, study group designation, education level, income, and study center; (b) within-group and group-collapsed trend tests of categorical 25OHD concentration effects on preeclampsia development were applied. Finally, we evaluated the dose-response association between maternal vitamin $\mathrm{D}$ serum concentrations at 10 to 18 weeks of gestation and risk of preeclampsia after adjustment for the informative variables identified in the primary outcome analysis (Table 2). For all of the ITT analyses, 2 -sided $P$ values of less than 0.05 were considered statistically significant.

Transcriptomic variations related to preeclampsia. SV analyses were conducted, and tests for differential gene expression included an adjustment for expression heterogeneity. Differential expression analysis was carried out using the Bioconductor "RankProd" package, which implements the rank product method for identifying differentially expressed genes (48). Differentially expressed (up- or downregulated) genes were identified through permutation analysis, after setting the percentage of the false prediction threshold for which the FDR was less than 0.05. The number of permutations used was set as the package default value. We separately identified genes associated with high/low vitamin D status (at a cutoff of $30 \mathrm{ng} / \mathrm{ml}$ ) and preeclampsia case status and then identified the overlap of these 2 gene modules. We replicated the overlap in our second case-control study using the rank product method. Those genes having a $P$ value of less than 0.05 in the replication population were considered for further investigation in the network and pathway analysis as well as for literature curation for their relationship to preeclampsia.

In addition to the GeneCards (53) and MetaCore databases (https://lsresearch.thomsonreuters.com/pages/solutions/1/metacore) (overall 673 annotations), the replicated gene signatures were manually curated in order to validate their connection to preeclampsia according to the scientific literature and previous preeclampsia research. Using the Human Protein Atlas (HPA), the subset of replicated signatures with evidence of tissue-specific expression in placenta was identified (54). Figures 3 and 4 provide an overview of our approach and a summary of the results.

Molecular and functional interaction gene networks. The connectivity of the replicated signatures was explored by mapping onto functional protein-protein interaction networks from HumanNet v.1 (55). The identified gene set (preeclampsia module) was subsequently used to construct the "observable preeclampsia module," defined as the largest connected component (LCC) of the replicated signature genes (17). Thereafter, the connectivity of this module to the known vitamin Dand IL-10-signaling pathways using available data from the National Cancer Institute's (NCI's) Pathway Interaction Database (http://pid. nci.nih.gov) was explored. GO enrichment analysis was conducted to investigate the biological processes involved in the identified modules (56). Further details on the methods are provided in Figure 2.

Molecular interaction networks and pathway enrichment analysis. To understand the functional relationships underlying the set of replicated differentially expressed genes, we explored their connectivity in the HumanNet functional network (55). HumanNet uses a naive Bayes approach to weight different types of evidence together into a single interaction score focusing on data collected from humans, yeast, worms, and flies. We mapped the differentially expressed genes encoding proteins in the interactome and identified the LCC (or "observable preeclampsia module," Figure 5) (17). Furthermore, we tested the closeness of the observable preeclampsia module to the vitamin D and IL-10 pathways, as described below. Ordered functional enrichment for the representative GO terms "biological processed," "molecular function," and "cell component" annotations for both the replicated gene set and observable preeclampsia module was carried out with g:Profiler $(56,57)$. Accordingly, gene group functioning profiling was performed with default options limiting the output to significant results (multiple testing-corrected $P<0.05$ ).

Connectivity of preeclampsia module to vitamin D receptor and IL-1O pathways. We defined vitamin D and IL-10 pathways as reported in the NCI's Pathway Interaction and BioCarta databases, respectively (http://pid.nci.nih.gov). To test whether the observable preeclampsia module was connected to vitamin D and IL-10 pathways, we measured the closeness between the 2 sets of genes and compared this parameter with that having a random expectation of closeness (Figure 6). The assumption behind this exercise was that, even if the genes did not overlap between the preeclampsia module and vitamin D and IL-10 pathways, they should be localized in the same neighborhood 
of the interactome. The closeness was computed as the average of all possible shortest paths between 2 sets of genes, according to the following formula:

$$
\text { Avg dist }=\sum_{s \in P m, t \in P W} \frac{d(s, t)}{N_{P m} N_{P W}}
$$

(Equation 1)

In this formula, $d(s, t)$ is the shortest path from a gene $s$ in the preeclampsia module $(P m)$, and a gene $t$ of the interest pathway $(P w)$, and $N_{P m}$ and $N_{P w}$ denote the number of genes in each set. To determine the significance of this quantity, we selected $N_{P m}$ genes at random in the network and determined the average distance (Avg distrand) to the Pm. This exercise was repeated 1,000 times, and the significance was evaluated by computing the $Z$ score as follows:

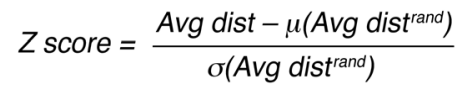

(Equation 2)

In this formula, $\mu$ (Avg dist $\left.{ }^{\text {rand }}\right)$ and $\sigma\left(A v g\right.$ dist $\left.{ }^{\text {rand }}\right)$ denote the mean value and $\mathrm{SD}$ of the random expectation. Any distance with a $Z$ score lower than -1.65 (i.e., modules closer than expected) was deemed significant; as such, a cutoff corresponded to $P \approx 0.05$ in the case of a normally distributed variable.

Study approval. The VDAART was approved by the IRBs of the participating institutions (Washington University in St. Louis, Boston Medical Center, Kaiser Health Care San Diego) and Brigham and Women's Hospital, and written consent was obtained from all participating pregnant women at their first enrollment visit. See the Supplemental material for the list of DSMB and DCC members (Supplemental Acknowledgments).

\section{Author contributions}

AAL, TFM, GO, ALP, RI, GM, RCS, LBB, RZ, BWH, NL, VC, and STW contributed to the design, coordination, and conduct of the trial. HM designed the project, performed statistical and bioinfor- matic analyses, and wrote the manuscript. DC, NL, VC, WQ, AS, MS, and SL assisted with data analyses. AS, MS, and SL assisted with the figures. AAL and STW supervised the trial. STW supervised the study project and manuscript preparation. TFM provided important advice on clinical aspects of the project and supervised the validation of the preeclampsia cases. JL provided important trial guidance and discussed the results. DAE and MAW provided the data from the OMEGA project, reviewed the data from the genomic experiments and contributed to the writing of the manuscript. DEH helped with the curation of the identified genes in the largest connected component, reviewed the data from the genomic experiments and contributed to the writing of the manuscript. All authors reviewed and assisted with editing the manuscript.

\section{Acknowledgments}

The authors thank the women who participated in this trial and all the study staff for their contributions to this research. The VDAART received support from the NHLBI (U01 HL091528, to STW and AAL, and R37 HL061795 and P50 GM107618, to JL) and from a National Research Service Award (NHLBI; T32-HL007427, to HM).

Role of trial sponsor: The National Heart, Lung, and Blood Institute (NHLBI) monitored the conduct of the trial and selected the membership of the Data and Safety Monitoring Board (DSMB). All communication between the investigators and the DSMB was handled through the staff of the NHLBI. All manuscripts, including the present one, during the course of the trial were presented to the DSMB for approval prior to submission for peer review. The NHLBI had no role in the design or conduct of the study, the collection, analysis, or interpretation of the data, or in the preparation, review, or approval of the manuscript, other than what pertained to the DSMB.

Address correspondence to: Scott T. Weiss, Professor of Medicine, Harvard Medical School Director, Partners HealthCare Personalized Medicine, Associate Director, Channing Division of Network Medicine 181 Longwood Avenue, Boston 02115, Massachusetts, USA. Phone: 617.525.2278; E-mail: scott.weiss@channing.harvard.edu
1. Wallis AB, Saftlas AF, Hsia J, Atrash HK. Secular trends in the rates of preeclampsia, eclampsia, and gestational hypertension, United States, 1987-2004. Am J Hypertens. 2008;21(5):521-526.

2. Jeyabalan A. Epidemiology of preeclampsia: impact of obesity. Nutr Rev. 2013;71(Suppl 1):S18-S25.

3. Aghajafari F, Nagulesapillai T, Ronksley PE, Tough SC, O’Beirne M, Rabi DM. Association between maternal serum 25-hydroxyvitamin D level and pregnancy and neonatal outcomes: systematic review and meta-analysis of observational studies. BMJ. 2013;346:f1169.

4. Tabesh M, Salehi-Abargouei A, Tabesh M, Esmaillzadeh A. Maternal vitamin D status and risk of pre-eclampsia: a systematic review and meta-analysis. JClin Endocrinol Metab. 2013;98(8):3165-3173.

5. Hyppönen E, et al. Vitamin D and pre-eclampsia: original data, systematic review and meta-analysis. Ann Nutr Metab. 2013;63(4):331-340.

6. Wei SQ, Qi HP, Luo ZC, Fraser WD. Maternal vitamin D status and adverse pregnancy outcomes: a systematic review and meta-analysis. JMatern Fetal Neonatal Med. 2013;26(9):889-899.

7. Pérez-López FR, et al. Effect of vitamin D supplementation during pregnancy on maternal and neonatal outcomes: a systematic review and meta-analysis of randomized controlled trials. Fertil Steril. 2015;103(5):1278-1288.e4.

8. Hollis BW, Wagner CL. Vitamin D and pregnancy: skeletal effects, nonskeletal effects, and birth outcomes. Calcif Tissue Int. 2013;92(2):128-139.

9. Allen RE, Rogozinska E, Cleverly K, Aquilina J, Thangaratinam S. Abnormal blood biomarkers in early pregnancy are associated with preeclampsia: a meta-analysis. Eur J Obstet Gynecol Reprod Biol. 2014;182:194-201.

10. Hossein-nezhad A, Spira A, Holick MF. Influence of vitamin D status and vitamin D3 supplementation on genome wide expression of white blood cells: a randomized double-blind clinical trial. PLOS ONE. 2013;8(3):e58725.

11. Enquobahrie DA, Williams MA, Qiu C, Siscovick DS, Sorensen TK. Global maternal early pregnancy peripheral blood mRNA and miRNA expression profiles according to plasma 25-hydroxyvitamin D concentrations. J Matern Fetal Neonatal Med. 2011;24(8):1002-1012.

12. Rajakumar A, et al. Maternal gene expression profiling during pregnancy and preeclampsia in human peripheral blood mononuclear cells. Placenta. 2011;32(1):70-78.

13. Sun CJ, Zhang L, Zhang WY. Gene expression profiling of maternal blood in early onset severe preeclampsia: identification of novel biomarkers. JPerinat Med. 2009;37(6):609-616.

14. Dahlstrøm B, Esbensen Y, Vollan H, Oian P, Bukholm G. Genome profiles in maternal blood during early onset preeclampsia and towards term. J Perinat Med. 2010;38(6):601-608.

15. Chaiworapongsa T, et al. Differences and similarities in the transcriptional profile of peripheral whole blood in early and late-onset preeclampsia: insights into the molecular basis of the phenotype of preeclampsiaa. J Perinat Med. 2013;41(5):485-504. 
16. Goh KI, Cusick ME, Valle D, Childs B, Vidal M, Barabási AL. The human disease network. Proc Natl Acad Sci U S A. 2007;104(21):8685-8690.

17. Menche J, et al. Disease networks. Uncovering disease-disease relationships through the incomplete interactome. Science. 2015;347(6224):1257601.

18. Asemi Z, Esmaillzadeh A. The effect of multi mineral-vitamin D supplementation on pregnancy outcomes in pregnant women at risk for pre-eclampsia. Int JPrev Med. 2015;6:62.

19. Sablok A, et al. Supplementation of vitamin $D$ in pregnancy and its correlation with feto-maternal outcome. Clin Endocrinol (Oxf). 2015;83(4):536-541.

20. Bodnar LM, Catov JM, Simhan HN, Holick MF, Powers RW, Roberts JM. Maternal vitamin D deficiency increases the risk of preeclampsia. JClin Endocrinol Metab. 2007;92(9):3517-3522.

21. Wei SQ, et al. Longitudinal vitamin D status in pregnancy and the risk of pre-eclampsia. BJOG. 2012;119(7):832-839.

22. Achkar M, et al. Vitamin D status in early pregnancy and risk of preeclampsia. Am JObstet Gynecol.2015;212(4):511.e1-511.e7.

23. Hollis BW, Johnson D, Hulsey TC, Ebeling M, Wagner CL. Vitamin D supplementation during pregnancy: double-blind, randomized clinical trial of safety and effectiveness. J Bone Miner Res. 2011;26(10):2341-2357.

24. Litonjua AA, et al. Effect of prenatal supplementation with vitamin $\mathrm{D}$ on asthma or recurrent wheezing in offspring by age 3 years: The VDAART Randomized Clinical Trial. JAMA. 2016;315(4):362-370.

25. Redman CW, Sargent IL. Latest advances in understanding preeclampsia. Science. 2005;308(5728):1592-1594.

26. Hennessy A, Pilmore HL, Simmons LA, Painter DM. A deficiency of placental IL-10 in preeclampsia. J Immunol. 1999;163(6):3491-3495.

27. Makris A, Xu B, Yu B, Thornton C, Hennessy A. Placental deficiency of interleukin-10 (IL-10) in preeclampsia and its relationship to an IL10 promoter polymorphism. Placenta. 2006;27(4-5):445-451.

28. Barrera D, Díaz L, Noyola-Martínez N, Halhali A. Vitamin D and inflammatory cytokines in healthy and preeclamptic pregnancies. Nutrients. 2015;7(8):6465-6490

29. Kalkunte S, Nevers T, Norris WE, Sharma S. Vascular IL-10: a protective role in preeclampsia. JReprod Immunol. 2011;88(2):165-169.

30. Lai Z, Kalkunte S, Sharma S. A critical role of interleukin-10 in modulating hypoxia-induced preeclampsia-like disease in mice. Hypertension.
2011;57(3):505-514.

31. Redman CW, Sargent IL. Immunology of pre-eclampsia. Am J Reprod Immunol. 2010;63(6):534-543.

32. Laresgoiti-Servitje E, Gómez-López N, Olson DM. An immunological insight into the origins of pre-eclampsia. Hum Reprod Update. 2010;16(5):510-524.

33. Goldman-Wohl D, Yagel S. Regulation of trophoblast invasion: from normal implantation to pre-eclampsia. Mol Cell Endocrinol. 2002; 187(1-2):233-238

34. Huppertz B. Placental origins of preeclampsia: challenging the current hypothesis. Hypertension 2008;51(4):970-975.

35. Kanasaki K, Kalluri R. The biology of preeclampsia. Kidney Int. 2009;76(8):831-837.

36. Rabaglino MB, Post Uiterweer ED, Jeyabalan A, Hogge WA, Conrad KP. Bioinformatics approach reveals evidence for impaired endometrial maturation before and during early pregnancy in women who developed preeclampsia. Hypertension. 2015;65(2):421-429.

37. Shin JS, Choi MY, Longtine MS, Nelson DM. Vitamin D effects on pregnancy and the placenta. Placenta. 2010;31(12):1027-1034.

38. Chan SY, et al. Vitamin D promotes human extravillous trophoblast invasion in vitro. Placenta. 2015;36(4):403-409.

39. De-Regil LM, Palacios C, Lombardo LK, Pena-Rosas JP. Vitamin D supplementation for women during pregnancy. Cochrane Database Syst Rev. 2016;(1):CD008873.

40. ACOG Committee on Obstetric Practice. ACOG Committee Opinion No. 495: Vitamin D: Screening and supplementation during pregnancy. Obstet Gynecol. 2011;118(1):197-198.

41. Appelgren KE, Nietert PJ, Hulsey TC, Hollis BW, Wagner CL. Analyzing adherence to prenatal supplement: does pill count measure up? Int J Endocrinol. 2010;2010:631971.

42. Litonjua AA, et al. The Vitamin D Antenatal Asthma Reduction Trial (VDAART): rationale, design, and methods of a randomized, controlled trial of vitamin $\mathrm{D}$ supplementation in pregnancy for the primary prevention of asthma and allergies in children. Contemp Clin Trials. 2014;38(1):37-50.

43. American College of Obstetricians Gynecologists' Task Force on Hypertension in Pregnancy. Hypertension in pregnancy. Report of the American College of Obstetricians and Gynecologists' Task Force on Hypertension in Pregnancy. Obstet Gynecol. 2013;122(5):1122-1131.

44. Farrell CJ, Martin S, McWhinney B, Straub I, Williams P, Herrmann M. State-of-the-art vitamin
D assays: a comparison of automated immunoassays with liquid chromatographytandem mass spectrometry methods. Clin Chem. 2012;58(3):531-542.

45. Denburg MR, et al. Comparison of two ELISA methods and mass spectrometry for measurement of vitamin D-binding protein: Implications for the assessment of bioavailable vitamin D concentrations across genotypes. J Bone Miner Res. 2016;31(6):1128-1136.

46. Bouillon R. Free or total 25OHD as marker for vitamin D status? J Bone Miner Res. 2016;31(6):1124-1127.

47. Bourgon R, Gentleman R, Huber W. Independent filtering increases detection power for high-throughput experiments. Proc Natl Acad Sci USA. 2010;107(21):9546-9551.

48. Breitling R, Armengaud P, Amtmann A, Herzyk P. Rank products: a simple, yet powerful, new method to detect differentially regulated genes in replicated microarray experiments. FEBS Lett. 2004;573(1-3):83-92.

49. Enquobahrie DA, Qiu C, Muhie SY, Williams MA. Maternal peripheral blood gene expression in early pregnancy and preeclampsia. Int J Mol Epidemiol Genet. 2011;2(1):78-94.

50. Thacher TD, Clarke BL. Vitamin D insufficiency. Mayo Clin Proc. 2011;86(1):50-60.

51. Saggese G, Baroncelli GI, Bertelloni S, Cipolloni C. Intact parathyroid hormone levels during pregnancy, in healthy term neonates and in hypocalcemic preterm infants. Acta Paediatr Scand. 1991;80(1):36-41.

52. Mulligan ML, Felton SK, Riek AE, BernalMizrachi C. Implications of vitamin D deficiency in pregnancy and lactation. Am JObstet Gynecol. 2010;202(5):429.e1-429.e9.

53. Safran M, et al. GeneCards Version 3: the human gene integrator. Database (Oxford) 2010;2010:baq020.

54. Pontén F, Schwenk JM, Asplund A, Edqvist $\mathrm{PH}$. The Human Protein Atlas as a proteomic resource for biomarker discovery. J Intern Med. 2011;270(5):428-446

55. Lee I, Blom UM, Wang PI, Shim JE, Marcotte EM. Prioritizing candidate disease genes by network-based boosting of genome-wide association data. Genome Res. 2011;21(7):1109-1121.

56. Ashburner M, et al. Gene ontology: tool for the unification of biology. The Gene Ontology Consortium. Nat Genet. 2000;25(1):25-29.

57. Reimand J, Arak T, Vilo J. g:Profiler--a web server for functional interpretation of gene lists (2011 update). Nucleic Acids Res. 2011;39(Web Server issue):W307-W315 